\title{
THE STRUCTURE OF BALANCED RINGS
}

\author{
By VLASTIMIL DLAB and CLAUS MICHAEL RINGEL†
}

[Received 6 August 1971-Revised 1 November 1971]

The present paper provides a complete description of left balanced rings. Recall that a ring is called left balanced if every left $R$-module $M$ is balanced, i.e. if, for any left $R$-module $M$, the natural homomorphism of $R$ to the double centralizer of $M$ is surjective. In [4], left balanced rings have been shown to be left artinian. Hence, by [7], a ring is left balanced if and only if it is a finite product of full matrix rings over local left balanced left artinian rings. Consequently, the study of left balanced rings is reduced to that of left artinian local rings.

Throughout the rest of the paper, $R$ denotes a local left artinian ring, $W$ is its radical, and $Q=R / W$ is the residue division ring. There is a unique simple left $R$-module ${ }_{R} Q$, and $E$ denotes its injective envelope (so $E$ is the only indecomposable injective left $R$-module). Then the main result of the present paper is the following:

Structure Theorem. $R$ is left balanced if and only if either (a) $R$ is uniserial or (b) $W^{2}=0,{ }_{R} W$ is simple, $W_{R}$ has length 2 , and $E$ has length 3, or (c) $W^{2}=0, W_{R}$ is simple, ${ }_{R} W$ has length 2 , and $E$ has length 2.

Here, $R$ is called uniserial, if all its left ideals and all its right ideals are linearly ordered by inclusion. The rings described under (b) and (c) will be called exceptional. As a matter of fact, it will be shown (Theorem 2.3) that a local ring $R$ satisfies the conditions (b) if and only if its opposite $R^{*}$ satisfies the condition (c). Consequently, an (arbitrary) ring is left balanced if and only if it is right balanced. As a by-product of our structural investigations in [4] and the present paper, a left artinian ring $A$ is shown to be balanced if and only if every finitely generated left $A$-module is balanced (Remark 3.7).

In this paper, we are concerned only with the necessity of the conditions. The sufficiency of (a) is well known and the sufficiency of (b) and (c) was essentially proved in [5].

If $Q=R / W$ is finitely generated over its centre, then $R$ is exceptional if and only if $W^{2}=0$ and $\partial_{R} W \times \partial W_{R}=2$ (Theorem 5.2). This is a consequence of the fact that a division subring of a division ring which is finitely generated over its centre, is of left index 2 if and only if it is

$\dagger$ Research supported by the National Research Council of Canada under Grant No. A-7257.

Proc. London Math. Soc. (3) 26 (1973) 446-462 
of right index 2. The existence of non-exceptional local rings satisfying the conditions $W^{2}=0$ and $\partial_{R} W \times \partial W_{R}=2$ is equivalent to the existence of a division ring with an isomorphic subring of right index 2 and left index different from 2 (Theorem 5.3).

Finally, a categorical characterization of balanced rings is given in Theorem 4.1: a left artinian ring $A$ is balanced if and only if the composition factors of each indecomposable left $A$-module are isomorphic, if every such $A$-module of length greater than 3 is uniserial, and any two indecomposable $A$-modules of a given length with isomorphic composition factors are isomorphic. In fact, a balanced ring has only finitely many isomorphism types of indecomposable modules (Theorem 4.2).

\section{Preliminaries}

Throughout the paper, the terminology and notation of [4] will be used as well as the notations $R, W, Q$, and $E$ fixed already. In particular, if $A$ is a ring with unity, $A^{*}$ denotes its opposite. By an $A$-module we always understand a unital $A$-module; the symbols ${ }_{A} M$ or $M_{A}$ will be used to underline the fact that $M$ is a left or a right $A$-module, respectively; $\partial M$ will denote the length of $M$. It should be noted that homomorphisms always act on the side opposite to that of the operators; in particular, every left $A$-module $M$ defines a right $\mathscr{C}$-module $M_{\mathscr{C}}$, where $\mathscr{C}$ is the centralizer of the $A$-module $M$.

We need three known results. In [4], we proved

Theorem 1.1. Let $R$ be left balanced. Then

either (i) $R$ is left uniserial,

or (ii) $W^{2}=0$, and $\partial_{R} W=2$,

or (iii) $W^{3}=0, \partial_{R}\left(W / W^{2}\right)=2$, and $W^{2}$ is the unique minimal left ideal.

Conversely, in [5], certain local rings with $W^{2}=0$ were shown to be left balanced. There, $Q$ was supposed to be commutative; however, this was used only to calculate the indecomposable injective modules. If we assume that $E$ has the appropriate properties, then $\S \S 3,4$, and 5 of [5] give the following two descriptions of $R$-modules (as well as proving that exceptional rings are balanced).

Theorem 1.2. Let $W^{2}=0,{ }_{R} W$ be simple, $W_{R}$ be of length 2 , and the injective envelope $E$ of ${ }_{R} Q$ be of length 3. Then

(a) every indecomposable left module is either simple, injective or isomorphic to ${ }_{R} R$, and every left module is a direct sum of these indecomposable modules;

(b) $R$ is left balanced. 
Theorem 1.3. Let $W^{2}=0, W_{R}$ be simple, ${ }_{R} W$ be of length 2 , and the injective envelope $E$ of ${ }_{R} Q$ be of length 2. Then

(a) every indecomposable left module is monogenic and either simple, injective, or isomorphic to ${ }_{R} R$, and every left module is a direct sum of these indecomposable modules;

(b) $R$ is left balanced.

In our investigations, the following two results on balanced modules will be required. We recall that an indecomposable $R$-module is said to have large kernels if, for every endomorphism $\varphi$ of $M$, either $\operatorname{Ker} \varphi=0$ or $\operatorname{Soc} M \subseteq \operatorname{Ker} \varphi$.

Lemma 1.4. Let $M$ be a balanced indecomposable left $R$-module of finite length and $m \in M$ such that $\operatorname{Ann}(m)=0$. Then

(i) denoting by $\mathscr{C}$ the centralizer of $M, m \mathscr{C}=M$, and

(ii) if, moreover, $M$ has large kernels, then $\operatorname{Soc} M \subseteq R m$.

Proof. Let $\mathscr{W}$ be the radical of $\mathscr{C}$. Since $M$ is of finite length, $\mathscr{W}$ is nilpotent and $M_{\mathscr{C}}$ has a non-trivial socle $\operatorname{Soc} M_{\mathscr{C}}$ and a non-trivial radical $M \mathscr{W}$ (see [1], Exercise 3, pp. 26-27). Now, $M /(m \mathscr{C}+M \mathscr{W}$ ) and Soc $M_{\mathscr{C}}$ are semisimple right $\mathscr{C}$-modules. Moreover, the local ring $\mathscr{C}$ has only one isomorphism type of simple modules, and therefore, if we show that any $\mathscr{C}$-homomorphism $\Psi$ of the form

$$
M_{\mathscr{C}} \stackrel{\varepsilon}{\longrightarrow} M /(m \mathscr{C}+M \mathscr{W}) \longrightarrow \operatorname{Soc} M_{\mathscr{C}} \stackrel{\iota}{\longrightarrow} M_{\mathscr{C}}
$$

(where $\varepsilon$ is the canonical epimorphism and $\iota$ the embedding) is trivial, then we have

$$
M=m \mathscr{C}+M \mathscr{W} .
$$

But $M$ is balanced, so $\Psi x=r x$ for some $r \in R$ and any $x \in M$. It follows from $r m=\Psi m=0$ and $A n n(m)=0$ that $r=0$; therefore $\Psi$ is trivial. The equality $M=m \mathscr{C}+M \mathscr{W}$ yields

$$
M=m \mathscr{C}+(m \mathscr{C}+M \mathscr{W}) \mathscr{W}=m \mathscr{C}+M \mathscr{W}^{2}
$$

and, by induction,

$$
M=m \mathscr{C}+M \mathscr{W}^{n} .
$$

Since $\mathscr{W}$ is nilpotent, $M=m \mathscr{C}$. This proves (i).

Since $M$ has large kernels, every element $\varphi \in \mathscr{W}$ satisfies $\left(\operatorname{Soc}_{R} M\right) \varphi=0$, and therefore $\operatorname{Soc}_{R} M \subseteq \operatorname{Soc} M_{\mathscr{C}}$. Also, $m \notin M \mathscr{W}$; otherwise $m \mathscr{C}$ would belong to $M \mathscr{W}$, but $M \mathscr{W} \neq M$. This implies that, for any element $x \in \operatorname{Soc}_{R} M$, we can find a $\mathscr{C}$-homomorphism $\Psi$ of the form

$$
M_{\mathscr{C}} \stackrel{\varepsilon^{\prime}}{\longrightarrow} M / M \mathscr{W} \longrightarrow \operatorname{Soc} M_{\mathscr{C}} \stackrel{\iota}{\longrightarrow} M_{\mathscr{C}}
$$


mapping $m$ onto $x$. But $\Psi$ is induced by left multiplication and so there is $r \in R$ with $r m=x$. The proof of Lemma 1.4 is complete.

The next result corresponds to Construction II of [4]; it implies that modules of equal length over a local left balanced, left artinian ring are isomorphic if they have simple socles.

Lemma 1.5. Let $M_{1}$ and $M_{2}$ be two faithful left $R$-modules such that, for $i \neq j$, every homomorphism $\varphi: M_{i} \rightarrow M_{j}$ satisfies $\left(\operatorname{Soc} M_{i}\right) \varphi=0$. Then $M_{1} \oplus M_{2}$ is not balanced.

Proof. We represent the elements of the centralizer of $M$ by matrices

$$
\left(\begin{array}{ll}
\varphi_{11} & \varphi_{12} \\
\varphi_{21} & \varphi_{22}
\end{array}\right), \quad \text { where } \varphi_{i j}: M_{i} \rightarrow M_{j} \text {. }
$$

Take a non-zero element $z$ of $\operatorname{Soc}_{R} R$, and define an additive homomorphism $\Psi: M \rightarrow M$ by

$$
\Psi\left(m_{1}, m_{2}\right)=\left(z m_{1}, 0\right) \quad \text { for }\left(m_{1}, m_{2}\right) \text { in } M_{1} \oplus M_{2} .
$$

Now, $z m_{i}$ belongs to Soc $M_{i}$; so, for $i \neq j$, we have

$$
z\left(m_{i} \varphi_{i j}\right)=\left(z m_{i}\right) \varphi_{i j}=0 .
$$

This implies that $\Psi$ belongs to the double centralizer of $M_{1} \oplus M_{2}$, because

$$
\begin{aligned}
{\left[\Psi\left(m_{1}, m_{2}\right)\right]\left(\begin{array}{ll}
\varphi_{11} & \varphi_{12} \\
\varphi_{21} & \varphi_{22}
\end{array}\right) } & =\left(z m_{1} \varphi_{11}, z m_{1} \varphi_{12}\right)=\left(z m_{1} \varphi_{11}+z m_{2} \varphi_{21}, 0\right) \\
& =\Psi\left[\left(m_{1}, m_{2}\right)\left(\begin{array}{ll}
\varphi_{11} & \varphi_{12} \\
\varphi_{21} & \varphi_{22}
\end{array}\right)\right]
\end{aligned}
$$

Assuming that $\Psi$ is induced by left multiplication by $\rho \in R$, the equation $\left(z m_{1}, 0\right)=\left(\rho m_{1}, \rho m_{2}\right)$ for all $m_{i} \in M_{i}$ implies $\rho=0$, because $M_{2}$ is faithful. But $z M_{1} \neq 0$, because $M_{1}$ is faithful. Lemma 1.5 follows.

\section{Exceptional rings}

The aim of $\S 2$ is to make a detailed study of the 'exceptional' rings defined in the Introduction to be those $R$ which satisfy conditions (b) or (c) of the Structure Theorem. We assume throughout $\S 2$ that $W^{2}=0$.

The division ring $Q=R / W$ operates on $W$ both from the left and the right and we get two vector spaces ${ }_{Q} W$ and $W_{Q}$. If $w$ is a non-zero element of $W$, we define a subring $S_{w}$ of $R$ by

$$
S_{w}=\{s \in R \mid s w \in w R\}
$$


Obviously, since $W w=0, W \subseteq S_{w}$. Moreover, if $s$ is a unit belonging to $S_{w}$, then the equality $s w=w r$ (for a suitable $r \in R$ ) implies $s^{-1} w=w r^{-1}$, and thus $s^{-1} \in S_{w}$ as well. Consequently, $S_{w} / W$ is a division subring of $Q=R / W$. If $\lambda$ is a unit of $R$, then

$$
S_{\lambda w}=\{s \in R \mid s \lambda w \in \lambda w R\}=\left\{s \in R \mid \lambda^{-1} s \lambda w \in w R\right\}=\lambda S_{w} \lambda^{-1}
$$

and thus, in particular,

$$
\operatorname{dim}_{\left(S_{w} / W\right)} Q=\operatorname{dim}_{\left(S_{\lambda w} / W\right)} Q .
$$

Similarly, we define the subring $T_{w}$ of $R$ :

$$
T_{w}=\{t \in R \mid w t \in R w\}
$$

Again, $W \subseteq T_{w}, T_{w} / W$ is a division subring of $Q$, and, for an arbitrary unit $\rho$ of $R$,

$$
\operatorname{dim} Q_{\left(T_{w} / W\right)}=\operatorname{dim} Q_{\left(T_{w \rho} / W\right)} .
$$

Proposition 2.1. Let $\operatorname{dim}_{Q} W=1$ and $\operatorname{dim} W_{Q}=2$. Then the following statements are equivalent:

(i) $\operatorname{dim}_{\left(S_{w} / W\right)} Q=2$;

(ii) there exist two linearly independent elements $v, w$ of $W_{Q}$ such that $W=w R+S_{w} v$

(iii) $R$ is exceptional.

Proof. In order to prove that (i) implies (ii), let $w$ be a non-zero element of $W, S=S_{w}$, and let $\operatorname{dim}_{(S / W)} Q=2$. Thus, there exists $r \in R \backslash S$ such that $R=S+S r+W$. Putting $v=r w$, one gets

$$
W=R w=(S+S r+W) w=S w+S v=w R+S v,
$$

because $\operatorname{dim}_{Q} W=1$ implies $S w=w R$.

Now, to establish that (ii) implies (iii), we are going to show that

$$
M={ }_{R}(R \oplus R) / D, \quad \text { where } D=\{(\lambda v, \lambda w) \mid \lambda \in R\},
$$

is an indecomposable injective left $R$-module, hence a copy of $E$. To prove injectivity of $M$ assume that a homomorphism $\varphi:{ }_{R} W \rightarrow M$ is given; we are required to extend it to a homomorphism from ${ }_{R} R$ to $M$. Obviously, $\varphi$ is determined by the image of $w$; and, since $w \varphi \in W M$, we can find $w_{1}, w_{2} \in W$ such that

$$
w \varphi=\left(w_{1}, w_{2}\right)+D
$$

But, $w_{2}=\lambda w$ and thus, for some $w_{0} \in W$,

$$
\left(w_{1}, w_{2}\right)+D=\left(w_{1}-\lambda v, 0\right)+(\lambda v, \lambda w)+D=\left(w_{0}, 0\right)+D .
$$

Now $w_{0} \in w R+S_{w} v$, and therefore there are elements $r_{1} \in R, s \in S_{w}$, and $r_{2} \in R$ such that

$$
w_{0}=w r_{1}+s v \text { and } s w=-w r_{2} .
$$


We claim that the homomorphism

$$
{ }_{R} R{\stackrel{\left(r_{1}, r_{2}\right)}{\longrightarrow}}_{R}(R \oplus R) \stackrel{\varepsilon}{\longrightarrow} M,
$$

where $\varepsilon$ is the canonical epimorphism, is an extension of $\varphi$. Indeed, the element $w$ is mapped into

$$
w\left(r_{1}, r_{2}\right)+D=\left(w_{0}-s v,-s w\right)+D=\left(w_{0}, 0\right)+D=w \varphi,
$$

as required. Consequently, $M$ is injective and, being of length 3 , necessarily indecomposable. So $E$ also has length 3 .

To complete the proof, let us verify that (iii) implies (i). An indecomposable injective left $R$-module $M$ of length 3 is necessarily an amalgam of two copies of ${ }_{R} R$ over its socle. Thus,

$$
M={ }_{R}(R \oplus R) / D \quad \text { with } D=\{(\lambda v, \lambda w) \mid \lambda \in R\}
$$

for suitable $v$ and $w$ of $W$. Now, take an arbitrary $x \in W$ and consider the homomorphism $\varphi:{ }_{R} W \rightarrow M$ mapping $w$ into $(x, 0)+D$. Extend $\varphi$ to a homomorphism from ${ }_{R} R$ to $M$ and lift the latter to

Hence,

$$
{ }_{R} R \stackrel{\left(r_{1}, r_{2}\right)}{\longrightarrow}{ }_{R}(R \oplus R) \text {. }
$$

and thus

$$
\left(w r_{1}, w r_{2}\right)-(x, 0) \in D \text {, }
$$

$$
\left(w r_{1}-x, w r_{2}\right)=(\lambda v, \lambda w) \text { for some } \lambda \in R \text {. }
$$

Therefore, writing $S_{w}=S$,

Thus

$$
\lambda \in S \text { and } x=w r_{1}-\lambda v \in w R+S v .
$$

$$
R w=W=w R+S v=S w+S v=(S+S r) w
$$

with a suitable $r \in R$, and hence

$$
R=S+S r+W
$$

Now, $r \notin S$; for, otherwise $W=w R+S r w=w R$ in contradiction to $\operatorname{dim} W_{Q}=2$. As a consequence, $\operatorname{dim}_{(S / W)} Q=2$, as required. The proof of Proposition 2.1 is complete.

Proposition 2.2. Let $\operatorname{dim}_{Q} W=2$ and $W_{Q}=1$. Then the following statements are equivalent:

(i) $\operatorname{dim} Q_{\left(T_{w} / W\right)}=2$;

(ii) there exist two linearly independent elements $v, w$ of ${ }_{Q} W$ such that

(iii) $R$ is exceptional.

$$
W=R w+v T_{w}
$$

Proof. Both statements (i) and (ii) are dual to those of Proposition 2.1, and thus they are equivalent. In order to show that (ii) implies (iii), 
let $v, w$ be the elements given in (ii). We are going to prove that $R / R w$ is an indecomposable injective $R$-module, hence a copy of $E$. To this end, let $\varphi:{ }_{R} W \rightarrow R / R w$ be a non-zero homomorphism. Since right multiplication by elements of $R$ is transitive on $W$, we can evidently assume that $\operatorname{Ker} \varphi=R w$. Thus, $\varphi$ is determined by the conditions $w \varphi=0$ and $v \varphi=w_{0}+R w$ for a suitable $w_{0} \in W$. In view of the relation $W=R w+v T_{w}$, we have

$$
w_{0}=r w+v t \quad \text { for some } r \in R \text { and } t \in T_{w} .
$$

Consequently, the homomorphism

$$
{ }_{R} R \stackrel{t}{\longrightarrow}{ }_{R} R \stackrel{\varepsilon}{\longrightarrow} R / R w
$$

maps $R w$ into $0, v$ into $w_{0}-r w+R w=w_{0}+R w$, and is thus an extension of $\varphi$ to ${ }_{R} R$. Therefore $R / R w$ is injective. It is clearly indecomposable, and clearly of length 2 . So $E$ also has length 2 .

Finally, we verify that (iii) implies (i). Let $M$ be an indecomposable injective left $R$-module of length 2 ; hence $M \cong R / R w$ for some non-zero element $w \in W$. Let $v \in{ }_{Q} W$ so that $v$ and $w$ are linearly independent. Take an arbitrary element $x \in W$ and consider the homomorphism $\varphi:{ }_{R} W \rightarrow M$ such that

$$
v \varphi=x+R w \text { and } w \varphi=0 .
$$

Since $M$ is injective, $\varphi$ can be extended to a homomorphism from ${ }_{R} R$ to $M$, and therefore lifted to ${ }_{R} R \stackrel{r}{\longrightarrow}{ }_{R} R$. From here, it follows that $w r \in R w$ and thus $r \in T_{w}$; moreover, $x-v r \in R w$. Consequently,

$$
W=R w+v T_{w}
$$

which fact completes the proof of Proposition 2.2.

Theorem 2.3. A ring $R$ is exceptional if and only if its opposite $R^{*}$ is exceptional.

Proof. This follows immediately from the fact that a ring satisfies the conditions of Proposition 2.1 (i) if and only if its opposite satisfies the conditions of Proposition 2.2 (i).

We conclude this section by remarking that the existence of exceptional rings will be shown in $\S 5$. There we shall also consider the conditions under which a local ring $R$ with

$$
W^{2}=0 \text { and } \operatorname{dim}_{Q} W \times \operatorname{dim} W_{Q}=2
$$

is exceptional. It will be shown that this question is equivalent to deciding whether certain division subrings of $Q$ have left index equal to right index. 


\section{The structure theorem}

First, we give a complete description of left balanced local rings $R$ with $W^{2}=0$.

Lemma 3.1. Let $W^{2}=0, \operatorname{dim}_{Q} W=1$, and $\operatorname{dim} W_{Q} \geqslant 3$. Let $u, v, w$ be linearly independent in $W_{Q}$. Then

$$
M={ }_{R}(R \oplus R \oplus R) / D, \text { where } D=\{(\lambda u, \lambda v, \lambda w) \mid \lambda \in R\},
$$

is an indecomposable left $R$-module with large kernels.

Proof. First, there is no homomorphism of $M$ onto ${ }_{R} R$. For, assuming the converse, we get a homomorphism

$$
\left(\begin{array}{l}
r_{1} \\
r_{2} \\
r_{3}
\end{array}\right):{ }_{R}(R \oplus R \oplus R) \rightarrow{ }_{R} R
$$

such that $D$ is mapped into 0 . Thus $u r_{1}+v r_{2}+w r_{3}=0$ and in view of the linear independence of $u, v, w$ in $W_{Q}$, all $r_{i} \in W$; therefore the homomorphism cannot be surjective.

Now, $\partial(\operatorname{Soc} M)=2$. For, assume $\partial(\operatorname{Soc} M)>2$, and consider the submodule $M^{\prime}={ }_{R}(R \oplus R \oplus W) / D$ of $M$. Obviously, $M^{\prime}$ is isomorphic to ${ }_{R}(R \oplus R)$, and therefore $\partial\left(\operatorname{Soc} M^{\prime}\right)=2$. By assumption, we can find an element $m \in \operatorname{Soc} M \backslash \operatorname{Soc} M^{\prime}$. Since $M^{\prime} \cap R m=0$ and

$$
\partial\left(M^{\prime} \oplus R m\right)=\partial\left(M^{\prime}\right)+1=5=\partial(M),
$$

we conclude that $M^{\prime}$ is a direct summand of $M$. But this contradicts the fact that $M$ has no epimorphic image isomorphic to ${ }_{R} R$.

Since Soc $M \supseteq\left[\operatorname{Soc}_{R}(R \oplus R \oplus R)\right] / D$ and since $\partial(\operatorname{Soc} M)=2$, we get

$$
\operatorname{Soc} M=\left[\operatorname{Soc}_{R}(R \oplus R \oplus R)\right] / D=\left[\operatorname{Rad}_{R}(R \oplus R \oplus R)\right] / D=\operatorname{Rad} M \text {. }
$$

Moreover, there is no indecomposable submodule of $M$ of length 3 . For, an indecomposable left $R$-module $N$ of length 3 has a simple socle, and thus $N$ would intersect trivially either $M_{1}=R[(1,0,0)+D]$ or $M_{2}=R[(0,1,0)+D]$. Assuming $N \cap M_{1}=0$, we conclude, in view of $\partial M_{1}=2$ and $\partial M=5$, that $M_{1}$ is a direct summand of $M$. But, $M_{1}$ is obviously isomorphic to ${ }_{R} R$, and this contradicts the fact that $M$ has no epimorphic image isomorphic to ${ }_{R} R$. Therefore $N \cap M_{1} \neq 0$. Similarly $N \cap M_{2} \neq 0$. Therefore no such $N$ can exist. Also, there cannot be an indecomposable submodule of $M$ of length 4 . For, such a submodule would have a simple socle and thus contain an indecomposable submodule of length 3 .

Finally, since $M$ has no indecomposable epimorphic image of length 2 and no indecomposable submodules of length 3 or 4, the image of every 
proper endomorphism $\varphi$ of $M$ is semisimple and therefore $(\operatorname{Rad} M) \varphi=0$; consequently, (Soc $M) \varphi=0$ and so $M$ is an indecomposable module with large kernels.

Lemma 3.2. Let $R$ be left balanced, $W^{2}=0$, and $\operatorname{dim}_{Q} W=1$. Then $R$ is uniserial or exceptional.

Proof. Assuming that $R$ is left balanced, we want first to show that $\operatorname{dim} W_{Q} \leqslant 2$. Otherwise, there are three linearly independent elements $u, v, w$ in $W_{Q}$, and according to Lemma 3.1,

$$
M={ }_{R}(R \oplus R \oplus R) / D, \quad \text { where } D=\{(\lambda u, \lambda v, \lambda w) \mid \lambda \in R\},
$$

is an indecomposable left $R$-module with large kernels. Since, obviously, $\operatorname{Ann}[(1,0,0)+D]=0$, it follows from Lemma 1.4 (ii) that

$$
\text { Soc } M \subseteq R[(1,0,0)+D] \text {. }
$$

Taking $(0, u, 0)+D \in \operatorname{Soc} M$, we get $\left(r_{0}, 0,0\right)+D=(0, u, 0)+D$ for a suitable $r_{0} \in R$, and thus $\left(r_{0},-u, 0\right) \in D$. This is impossible, and therefore $\operatorname{dim} W_{Q} \leqslant 2$.

If $\operatorname{dim} W_{Q}=1, R$ is uniserial. Therefore, we assume that $\operatorname{dim} W_{Q}=2$. In order to prove that $R$ is exceptional it is sufficient to show, in view of Proposition 2.1, that $W=w R+S_{w} v$ for two linearly independent elements $v$ and $w$ of $W_{Q}$. Since the $R$-module

$$
N={ }_{R}(R \oplus R) / D \quad \text { with } D=\{(\lambda v, \lambda w) \mid \lambda \in R\}
$$

is indecomposable and since, obviously,

$$
\operatorname{Ann}[(1,0)+D]=0, \quad N=[(1,0)+D] \mathscr{C},
$$

by Lemma 1.4 (i). Therefore, taking an arbitrary $r \in R$, there is $\varphi \in \mathscr{C}$ such that

Lifting $\varphi$ to

$$
[(1,0)+D] \varphi=(r, 0)+D .
$$

$$
\left(\begin{array}{ll}
\alpha_{11} & \alpha_{12} \\
\alpha_{21} & \alpha_{22}
\end{array}\right):{ }_{R}(R \oplus R) \rightarrow{ }_{R}(R \oplus R),
$$

we find that $\left(\alpha_{11}-r, \alpha_{12}\right) \in D$ and, in particular, $\alpha_{11}-r \in W$ and $\alpha_{12} \in W$. Also, applying this homomorphism to $(v, w) \in D$, we obtain

Hence,

$$
\left(v \alpha_{11}+w \alpha_{21}, v \alpha_{12}+w \alpha_{22}\right)=\left(v r+w \alpha_{21}, w \alpha_{22}\right) \in D .
$$

$$
v r+w \alpha_{21}=\lambda v \text { and } \quad w \alpha_{22}=\lambda w \quad \text { for some } \lambda \in R .
$$

Therefore $\lambda \in S_{w}$ and $v r \in w R+S_{w} v$. Consequently

as required.

$$
W=v R+w R \subseteq W r+S_{w} v,
$$


Lemma 3.3. Let $W^{2}=0, \operatorname{dim}_{Q} W=2$, and $\operatorname{dim} W_{Q} \geqslant 2$. Let $u, v$ be linearly independent elements in $W_{Q}$. Then

$$
M={ }_{R}(R \oplus R) / D, \text { where } D=\{(\lambda u, \lambda v) \mid \lambda \in R\},
$$

is an indecomposable left $R$-module with large kernels.

Proof. First, let $\varphi: M \rightarrow{ }_{R} R$. Then, lifting $\varphi$ to $\left(\begin{array}{l}r_{1} \\ r_{2}\end{array}\right):{ }_{R}(R+R) \rightarrow{ }_{R} R$ mapping $D$ to 0 , we find that $u r_{1}+v r_{2}=0$. In view of the linear independence of $u, v$ in $W_{Q}, r_{1}$ and $r_{2}$ belong to $W$ and hence $\varphi$ is not surjective. As an immediate consequence, we have that $\partial(\operatorname{Soc} M)=3$. For, assume that $\partial(\operatorname{Soc} M)>3$ and consider the submodule $M^{\prime}=R[(1,0)+D]$ which is obviously isomorphic to ${ }_{R} R$. Since $\partial\left(\operatorname{Soc} M^{\prime}\right)=2$, we find a submodule $M^{\prime \prime}$ of Soc $M$ with $M^{\prime} \cap M^{\prime \prime}=0$ and $\partial M^{\prime \prime}=2$. But

$$
\partial\left(M^{\prime} \oplus M^{\prime \prime}\right)=\partial M^{\prime}+\partial M^{\prime \prime}=5=\partial M
$$

implies that $M^{\prime}$ is a direct summand of $M$. This contradicts the fact that $M$ has no epimorphic image isomorphic to ${ }_{R} R$. Since $\partial(\operatorname{Soc} M)=3$,

$$
\operatorname{Soc} M=\left[\operatorname{Soc}_{R}(R \oplus R)\right] / D=\left[\operatorname{Rad}_{R}(R \oplus R)\right] / D,
$$

and therefore $\operatorname{Soc} M=\operatorname{Rad} M$.

Now, $M$ contains no monogenic submodule of length 2. For, assume that $X$ is such a submodule. Again, since neither $R_{1}=R[(1,0)+D]$ nor $R_{2}=R[(0,1)+D]$ is a direct summand of $M, X$ necessarily contains the simple submodule $X^{\prime}=R[(u, 0)+D]=R[(0,-v)+D]=R_{1} \cap R_{2}$. Therefore the submodule $N=X+R_{1}$ satisfies $\partial(N / \operatorname{Rad} N)=2$ and $\partial N=4$. Since $\partial(M / N)=1, R_{2} \cong R$, and

$$
M / N=\left(R_{2}+N\right) / N \cong R_{2} /\left(R_{2} \cap N\right),
$$

it follows that $W R_{2}=R_{2} \cap N \subseteq N$. Hence $W R_{1}+W R_{2} \subseteq N$ and

$$
\partial(\operatorname{Soc} N)=3 \text {, }
$$

so that $N$ is a direct sum of $X$ and two copies of $Q$. This implies that $\partial(N / \operatorname{Rad} N)=3$, in contradiction to the formula $\partial(N / \operatorname{Rad} N)=2$ proved above. So no such $N$ exists.

Also, $M$ contains no indecomposable submodule of length 4. Assume that $Y$ is a submodule of $M$ of length 4. First, let $\partial(\operatorname{Soc} Y)=3$. Then $Y$ contains necessarily a copy of ${ }_{R} R$, so that $Y$ splits. Second, let $\partial(\operatorname{Soc} Y)=2$. Then, since $\partial(\operatorname{Soc} M)=3, M$ is isomorphic to $Y \oplus Q$, and this is obviously incompatible with the previously established formula $\operatorname{Rad} M=\operatorname{Soc} M$.

Now, it follows easily that each proper endomorphism of $M$ has semisimple image, hence kernel containing $\operatorname{Rad} M$. Since $\operatorname{Rad} M=\operatorname{Soc} M$, $M$ has large kernels and is indecomposable. 
Lemma 3.4. Let $R$ be left balanced, $W^{2}=0$, and $\operatorname{dim}_{Q} W=2$. Then $R$ is exceptional.

Proof. Assume that $R$ is left balanced. First, we shall prove that $\operatorname{dim} W_{Q}=1$. Assume the contrary and choose $v \in W, v \neq 0$. Since $\operatorname{dim}_{Q} W=2$ and $\operatorname{dim} W_{Q} \geqslant 2$, both $R v$ and $v R$ are proper subgroups of $W$ and therefore their set-theoretical union is a proper subset of $W$. It follows that there is an element $w \in W$ which is neither in $R v$ nor in $v R$. Consider the left $R$-module

$$
M={ }_{R}(R \oplus R) / D \quad \text { with } D=\{(\lambda v, \lambda w) \mid \lambda \in R\} .
$$

Since $v$ and $w$ are linearly independent in $W_{Q}, M$ is, according to Lemma 3.3, an indecomposable module with large kernels. Now, since $\operatorname{Ann}[(0,1)+D]=0$, we may apply Lemma 1.4 (ii) and find that Soc $M \subseteq R[(0,1)+D]$. Therefore, taking $(w, 0)+D \in \operatorname{Soc} M$, there is $r_{0} \in R$ such that $\left(w,-r_{0}\right) \in D$. Thus, in particular, $w=\lambda v$ for some $\lambda \in R$, in contradiction to $w \notin R v$.

Now, to complete the proof, we want to show that $W=R w+v T_{w}$. Consider the $R$-module $N=R / R w$; let $\mathscr{C}$ be its centralizer. Obviously, the rings $\mathscr{C}$ and $T_{w} / R w$ are isomorphic, and thus $\left(R w+v T_{w}\right) / R w$ is a non-zero $\mathscr{C}$-submodule of $N$. Since $\mathscr{C}$ is local, all simple $\mathscr{C}$-modules are isomorphic. Thus, the fact that $\operatorname{Rad} N_{\mathscr{C}} \neq N$ and $\operatorname{Soc} N_{\mathscr{C}}$ is essential in $N_{\mathscr{C}}$ (see again [1]) implies that there is a non-zero $\mathscr{C}$-homomorphism $\Psi: N \rightarrow N$ mapping $N$ into $\left(R w+v T_{w}\right) / R w$. Since $N$ is a balanced $R$-module, $\Psi$ is induced by the ring multiplication:

$\Psi n=r n$ for all $n \in N$ with a suitable non-zero $r \in R$.

Consequently,

$$
(r R+R w) / R w=\Psi(R / R w) \subseteq\left(R w+v T_{w}\right) / R w,
$$

and therefore $r R \subseteq R w+v T_{w}$. Since $\operatorname{dim} W_{Q}=1, W=R w+v T_{w}$, as required.

REMARK. Let us point out briefly that an alternative proof of the first part of Lemma 3.4 can run as follows: having proved that $\operatorname{dim} W_{Q}=1$, one can deduce from Lemma B of [2] that $\operatorname{dim} Q_{\left(T_{w} / W\right)} \leqslant 2$. By Proposition 2.2, the latter is equivalent to the fact that $R$ is exceptional.

We are ready to complete the proof of the Structure Theorem by showing:

Theorem 3.5. If $R$ is left balanced then it is either uniserial or exceptional.

Proof. Let $W$ be the radical of $R$ and $Q=R / W$. If $W^{2}=0$, it follows from (1.2) that $\operatorname{dim}_{Q} W \leqslant 2$. Therefore, by Lemmas 3.2 and 3.4, if $R$ is left balanced then it is uniserial or exceptional. 
Now, Theorem (3.5) would be established if we show that the only left balanced ring $R$ with $W^{2} \neq 0$ are the uniserial rings. Assume this is not so, that $R$ is left balanced, that $W^{2} \neq 0$, and that $R$ is not uniserial. Since the ring $R / W^{2}$ is left balanced, non-uniserial and the square of its radical is zero, it follows that $R / W^{2}$ is exceptional. Thus, by Theorem $(1,1)$, there are two remaining possibilities to exclude: (a) $W^{3}=0, \operatorname{dim}_{Q}\left(W / W^{2}\right)=2$ and $\operatorname{dim}_{Q} W^{2}=1$, and (b) $R$ is left uniserial but $\operatorname{dim}\left(W / W^{2}\right)_{Q}=2$.

Case (a). Assume that $W^{3}=0, \operatorname{dim}_{Q}\left(W / W^{2}\right)=2$ and $\operatorname{dim}_{Q} W^{2}=1$. Thus ${ }_{R} W$ is a module of length 3 with a simple socle. But ${ }_{R} W$ can be considered as a left $R / W^{2}$-module. Since $R / W^{2}$ is an exceptional ring with $\operatorname{dim}_{Q}\left(W / W^{2}\right)=2$, the indecomposable injective left $R / W^{2}$-module is of length 2 . Therefore no left $R / W^{2}$-module of length 3 has a simple socle.

Case (b). Assume that $R$ is left uniserial and that $\operatorname{dim}\left(W / W^{2}\right)_{Q}=2$. We may obviously assume $W^{3}=0$. In order to show that this case cannot occur, we shall construct two faithful non-isomorphic $R$-modules $M_{1}$ and $M_{2}$ with simple socles and use Lemma 1.5 to exhibit a nonbalanced $R$-module, namely $M_{1} \oplus M_{2}$.

First, since $S=R / W^{2}$ is exceptional, also the opposite of $S$ is exceptional, according to Theorem 2.3. Observing that ${ }_{R}\left(W / W^{2}\right)$ is simple, we can apply (1.3) (a) to the opposite of $S$ and conclude that every right $S$-module is a direct sum of monogenic modules. In particular, $W$ itself can be considered as a right $S$-module and Soc $W_{S}=\left(W^{2}\right)_{S}$. This follows from the fact that Soc $W_{S}$ as an ideal of $R$ is two-sided and cannot be equal to $W_{S}$, because $W^{2} \neq 0$. Now, since $W_{S}$ possesses a semisimple quotient $\left(W / W^{2}\right)_{S}$ of length 2 , there are, in view of the above decomposition, elements $x$ and $y$ in $W$ such that $x R \cap y R=0$, with $x+W^{2}, y+W^{2}$ linearly independent in $\left(W / W^{2}\right)_{S}$. Moreover, since $x$ and $y$ do not belong to $W^{2}=\operatorname{Soc} W_{R}, \partial(x R)=\partial(y R)=2$.

Now consider

$$
M_{1}={ }_{R}(R \oplus R) / D_{1}, \quad \text { where } D_{1}=\{(\lambda x, \lambda y) \mid \lambda \in R\} .
$$

Obviously, $\partial M_{1}=4$. The left $R$-module $M_{1}$ has no monogenic quotient of length 2. For, given a homomorphism $\varphi: M_{1} \rightarrow{ }_{R}\left(R / W^{2}\right)$, we can lift it to a homomorphism $\left(\begin{array}{l}r_{1} \\ r_{2}\end{array}\right):{ }_{R}(R \oplus R) \rightarrow{ }_{R} R$. Since $D_{1}$ is mapped into $W^{2}, x r_{1}+y r_{2}$ belongs necessarily to $W^{2}$. But $x+W^{2}$ and $y+W^{2}$ are linearly independent in $\left(W / W^{2}\right)_{R / W}$ and therefore both $x r_{1}$ and $y r_{2}$ belong to $W^{2}$. Consequently, both $r_{1}$ and $r_{2}$ lie in $W$ and hence $\varphi$ cannot be surjective. From here it follows easily that $\operatorname{Soc} M_{1}$ is simple. For, 
obviously, $N^{\prime}={ }_{R}(R \oplus W) / D_{1} \cong{ }_{R} R$ and thus $\partial\left(\operatorname{Soc} N^{\prime}\right)=1$; therefore, if $\partial\left(\operatorname{Soc} M_{1}\right) \geqslant 2$, then there exists a simple submodule $N^{\prime \prime} \subseteq M_{1}$ such that $N^{\prime} \cap N^{\prime \prime}=0$. Consequently,

$$
\partial\left(N^{\prime} \oplus N^{\prime \prime}\right)=\partial N^{\prime}+1=4=\partial M,
$$

and thus $M_{1}=N^{\prime} \oplus N^{\prime \prime}$. This contradicts the fact that there is no monogenic quotient of length 2.

To construct $M_{2}$, take a non-zero element $z \in y R \cap W^{2}$; such an element exists because $\partial(y R)=2$. Define the left $R$-module

$$
M_{2}={ }_{R}(R \oplus R) / D_{2}, \quad \text { where } D_{2}=\{(\lambda x, \lambda z) \mid \lambda \in R\} .
$$

Again $\partial M_{2}=4$. Also Soc $M_{2}$ is simple. For, if Soc $M_{2}$ is not simple, then an argument similar to the one given above shows that ${ }_{R}\left(W^{2} \oplus R\right) / D_{2} \cong{ }_{R} R$ is a direct summand of $M_{2}$. Thus $M_{2}$ possesses an epimorphic image which is a monogenic $R$-module of length 3. But a homomorphism $\varphi: M_{2} \rightarrow{ }_{R} R$ can be lifted to a homomorphism $\left(\begin{array}{l}r_{1} \\ r_{2}\end{array}\right):{ }_{R}(R \oplus R) \rightarrow{ }_{R} R$ mapping $D_{2}$ into 0 . Therefore, $x r_{1}+z r_{2}=0$. This relation shows that both $r_{1}$ and $r_{2}$ belong to $W$ and thus the homomorphism $\varphi$ cannot be surjective.

Now, $M_{1}$ and $M_{2}$ are two non-isomorphic left $R$-modules of length 4 . This follows from the fact that $M_{2}$ (in contrast to $M_{1}$ ) has a monogenic quotient of length 2, namely ${ }_{R}(R \oplus R) /\left(R \oplus W^{2}\right)$. Consequently, any homomorphism between $M_{1}$ and $M_{2}$ must have a non-trivial kernel. Since both Soc $M_{1}$ and Soc $M_{2}$ are simple, such a homomorphism $\varphi: M_{i} \rightarrow M_{j}$ (with $i \neq j$ ) satisfies $\left(\operatorname{Soc} M_{i}\right) \varphi=0$. Thus, applying Lemma 1.5 , we get a non-balanced left $R$-module. This contradiction shows that case (b) cannot occur.

The proof of Theorem (3.5), and therefore of the Structure Theorem, is now complete.

Since the concepts of a uniserial ring, as well as that of an exceptional ring, are self-dual, we have the following important result.

Corollary 3.6. A ring is left balanced if and only if it is right balanced.

From now on, we refer simply to a balanced ring.

REMARK 3.7. A left artinian ring $A$ is balanced if and only if every finitely generated left $A$-module is balanced.

Proof. If every finitely generated left $A$-module is balanced, then $A$ is a finite direct sum of full matrix rings over local rings $R_{i}$. Otherwise, it follows from [9] that there are non-balanced modules of length 2. Now, the property of being finitely generated is invariant under Morita equivalence, and so finitely generated $R_{i}$-modules are balanced (see, for 
example, [2]). Therefore it is sufficient to prove our theorem for local rings; and for these, the theorem holds because each non-balanced module used in the proofs in [4] and in the present paper is generated by at most four elements.

The assumption in Remark 3.7 that the ring $A$ be left artinian is essential. It is well known (see [1]) that every finitely generated abelian group is balanced, although the ring of all integers is not balanced.

\section{The module category of a balanced ring}

It is well known (and was used in the proof of the Structure Theorem) that the property of being balanced is invariant under Morita equivalence. Here, we characterize explicitly the balanced rings $A$ in terms of the module categories ${ }_{A} M$.

TheOREM 4.1. The left artinian ring $A$ is balanced if and only if the category ${ }_{A} M$ of all left $A$-modules has the following properties:

(i) the composition factors of each indecomposable left $A$-module are isomorphic,

(ii) every indecomposable left $A$-module with length greater than 3 is uniserial, and

(iii) any two indecomposable left $A$-modules of a given length and with isomorphic composition factors are isomorphic.

Proof. First, note that $A$ is the direct sum of full matrix rings over local rings $R_{i}$ if and only if condition (i) is satisfied in ${ }_{A} M$. Then, ${ }_{A} M$ satisfies (ii) and (iii) if and only if, for all $i$, the categories ${ }_{R_{i}} M$ satisfy these conditions. Therefore, it is sufficient to prove Theorem 4.1 for a local left artinian ring $A=R$.

The necessity of the conditions follows immediately from Theorem 3.5 together with Theorems (1.2) and (1.3).

In order to prove the sufficiency, let us first assume that all indecomposable left $R$-modules are uniserial. Then $R$ is trivially left uniserial. Also, $R$ is right uniserial. For otherwise ${ }_{R}(R \oplus R) / D$, where $D=\{(\lambda u, \lambda v) \mid \lambda \in R\}+\left(W^{2} \oplus W^{2}\right)$ with linearly independent elements $u, v$ in $\left(W / W^{2}\right)_{Q}$, is a non-uniserial indecomposable left $R$-module.

If there is a non-uniserial indecomposable $R$-module $X$ of length 3 with a simple socle, then $X$ is necessarily injective and $R$ (being a monogenic indecomposable $R$-module) is left uniserial. Consequently, ${ }_{R} R$ can be embedded in $X$ and therefore $W^{2}=0$. By Lemma 3.1, $\operatorname{dim} W_{Q}=2$; for, otherwise, there would exist an indecomposable nonuniserial $R$-module of length 5 . Hence, $R$ is exceptional and thus balanced. 
If there is a non-uniserial indecomposable $R$-module $Y$ of length 3 with a non-simple socle, then $Y / \operatorname{Rad} Y$ is simple and thus, necessarily, $Y \cong{ }_{R} R$. Consequently, $W^{2}=0, \operatorname{dim}_{Q} W=2$ and the indecomposable injective is uniserial (and of length 2). By Lemma 3.3, $\operatorname{dim} W_{Q}=1$; hence, $R$ is again exceptional and therefore balanced.

Theorem 4.2. A balanced ring has only finitely many isomorphism types of indecomposable modules.

Proof. A balanced ring is left artinian, therefore the length of the uniserial left $A$-modules is bounded. For any simple module $S$, all indecomposable left $A$-modules with composition factors isomorphic to $S$ are uniserial or of length 3, and any two of them are isomorphic, if their lengths are equal. This follows from Theorem 4.1. Since there is only a finite number of non-isomorphic simple $A$-modules, the number of isomorphism types of indecomposable left modules is finite. By duality, also the number of isomorphism types of indecomposable right modules is finite.

\section{The residue division ring}

Recall that $R$ denotes a local left artinian ring, $W$ is its radical, and $Q=R / W$ is the residue division ring. In this section we seek conditions under which such a ring, with

$$
W^{2}=0 \text { and } \partial_{R} W \times \partial W_{R}=2,
$$

is exceptional. It will be shown that this question is equivalent to deciding whether certain division subrings of $Q$ have left index equal to right index (cf. [10]).

We may restrict to the case $\partial_{R} W=1$. We start with the following lemma which calculates the right index of $S_{w} / W$ in $Q$.

LEMMA 5.1. Let $W^{2}=0$ and $\operatorname{dim}_{Q} W=1$. Let $w$ be a non-zero element of $W$. Then $S_{w} / W$ is isomorphic to $Q$ and

$$
\operatorname{dim} Q_{\left(S_{v} / W\right)}=\partial W_{R} .
$$

Proof. Write $S=S_{w}$ and define a morphism $\alpha: Q \rightarrow W$ by

$$
\alpha(r+W)=r w \text { for } r \in R .
$$

Furthermore, define a morphism $\beta: S / W \rightarrow Q$ by

$$
\beta(s+W)=t+W \text { for } s \in S \text { and } t \in R \text { such that } s w=w t .
$$

Then $\alpha$ and $\beta$ are well-defined bijections because $\operatorname{Ann}(w)=W$ and $R w=W$. Clearly, $\alpha$ and $\beta$ are additive and $\beta$ is easily verified to be multiplicative. Therefore $\beta$ is a ring isomorphism. Now, the pair 
$(\alpha, \beta): Q \times S / W \rightarrow W \times Q$ satisfies, for any $r \in R, s \in S$, and $t \in R$ with $s w=w t$,

$$
\begin{aligned}
\alpha[(r+W)(s+W)] & =\alpha(r s+W)=r s w=r w t=r w(t+W) \\
& =\alpha(r+W) \beta(s+W),
\end{aligned}
$$

which implies the equation $\operatorname{dim} Q_{S / W}=\operatorname{dim} W_{Q}=\partial W_{R}$.

If $R$ is a local ring with

$$
W^{2}=0, \quad \partial_{R} W=1 \quad \text { and } \quad \partial W_{R}=2,
$$

then Lemma 5.1 shows that $\operatorname{dim} Q_{S_{w} / W}=2$. But $R$ is exceptional if and only if $\operatorname{dim} Q_{S_{w} / W}=2$. Therefore, if $Q$ has the property that any division subring of right index 2, which is isomorphic to $Q$, has also left index 2, then the above conditions imply that $R$ is exceptional. In particular, we have the following theorem generalizing the result of [6].

Theorem 5.2. Assume that $R / W$ is finitely generated over its centre. Then $R$ is balanced if and only if it is uniserial or

$$
W^{2}=0 \text { and } \partial_{R} W \times \partial W_{R}=2 .
$$

Proof. If $R / W$ is finite-dimensional over its centre, then a division subring of $Q=R / W$ has right index 2 if and only if it has left index 2 ([8], p. 158). So $R$ is exceptional if and only if $W^{2}=0$ and $\partial_{R} W \times \partial W_{R}=2$.

The existence of a local ring $R$, with $W^{2}=0$ and $\partial_{R} W \times \partial W_{R}=2$, which is not exceptional would imply that the concept of a balanced ring involves not only the structure of the lattices of left and of right ideals, but also the embeddings of certain subrings.

THEOREM 5.3. The following assertions are equivalent.

(i) There exists a local ring $R$ with $W^{2}=0$ and $\partial_{R} W \times \partial W_{R}=2$, which is not exceptional.

(ii) There exists a division ring $D$ with a subring $D^{\prime}$ of right index 2 and of left index $\neq 2$, such that $D$ and $D^{\prime}$ are isomorphic.

Proof. In order to show that (i) implies (ii), we may assume that the given local ring $R$ satisfies $\partial_{R} W=1$. Otherwise, we consider the dual ring. Let $D=Q$, and $D^{\prime}=S_{w} / W$ for some non-zero element $w$ of $W$. Then, according to Lemma 5.1, $D$ and $D^{\prime}$ are isomorphic and $\operatorname{dim} D_{D^{\prime}}=2$; but because $R$ is not exceptional, $\operatorname{dim}_{D^{\prime}} D \neq 2$.

Conversely, let $D^{\prime}$ be a subring of the division ring $D$ such that there exists an isomorphism $\gamma: D \rightarrow D^{\prime}$. We form the ring $R$ of all pairs $(a, b)$ of elements of $D$ with component-wise addition and the following multiplication

$$
(a, b)\left(a^{\prime}, b^{\prime}\right)=\left(a a^{\prime}, a b^{\prime}+b \gamma(a)\right)
$$


Then the set of all elements $(0, b)$, with $b \in D$, is the radical $W$ of $R$. The ring $R$ is local, $W^{2}=0$, and $\partial_{R} W=1$. Let $w=(0,1)$. Then $(a, b)$ belongs to $S_{w}$ if and only if $a \in D^{\prime}$. Therefore, $\partial W_{R}=\operatorname{dim} D_{D^{\prime}}=2$, according to Lemma 5.I. But $\operatorname{dim}_{\left(S_{w} / W\right)} Q \neq 2$, so $R$ is not exceptional.

REMarK 5.4. P. M. Cohn has constructed in [3] an example of a division ring $D$ with a division subring $D^{\prime}$ of right index 2, but of left index different from 2. Thus, the question is whether such a subring $D^{\prime}$ exists which is, in addition, isomorphic to $D$.

The authors are indebted to the referee for valuable suggestions concerning the final form of the manuscript.

\title{
REFERENCES
}

1. N. BouRbakI, Algèbre, Chapitre VIII (Hermann, Paris, 1958).

2. V. P. CAMILLO and K. R. FuLLER, 'Balanced and QF-1 algebras', to appear.

3. P. M. Corr, 'Quadratic extensions of skew fields', Proc. London Math. Soc. 11 (1961) 531-56.

4. V. Dlab and C. M. Ringex, 'Rings with the double centralizer property', J. Algebra 22 (1972) 480-501.

5. —— 'On a class of balanced non-uniserial rings', Math. Ann. 195 (1972) 279-91.

6. _ 'Balanced local rings with commutative residue fields', Bull. Amer. Math. Soc. 78 (1972) 771-74.

7. K. R. FUlLer, 'Primary rings and double centralizers', Pacific J. Math. 34 (1970) 379-83.

8. N. JACOBSON, Structure of rings (Coll. Publ. Amer. Math. Soc., Vol. 37, 1956).

9. J. P. JANS, 'On the double centralizer condition', Math. Ann. 188 (1970) 85-89.

10. A. Rosenberg and D. Zelinsky, 'Finiteness of the injective hull', Math. $Z$. 70 (1959) 372-80.

\author{
Carleton University \\ Ottawa 1 \\ Ontario \\ Canada
}

